\title{
Impacto do Hipotireoidismo Subclínico na Resposta Cárdio- Pulmonar em Esforço e na Recuperação
}

\begin{abstract}
RESUMO
No intuito de identificar as manifestações clínicas do hipotireoidismo subclínico (HS) durante o estresse físico e na recuperação, foram comparadas 15 portadoras de HS com 16 mulheres saudáveis através de um teste cárdio-pulmonar em esteira ergométrica. Análise das médias obtidas através do Mann-Whitney U Test. As pacientes obtiveram valores menores no pico do exercício para fração expirada de $\mathrm{O}_{2}(14,90 \pm 1,05 \times 16$ $\pm 1,14 \% ; p=0,014)$; na variação da pressão arterial sistólica $(34,33 \pm 17,92$ $x 52,50 \pm 17,22 ; p=0,009)$; na duração do exercício $(8,83 \pm 2,91 \times 14,5 \pm 5,63$ $\min ; \mathrm{p}=0,0005)$, na carga máxima de teste $(11,6 \pm 4,22 \times 18,94 \pm 5,45 \%$; $p=0,0004$ ), além de tendências na razão de trocas gasosas e na freqüência cardíaca de pico. Entre o primeiro e o terceiro minutos de recuperação, houve uma redução média de $0,71 \mathrm{mmHg}$ na pressão arterial diastólica para essas pacientes, comparado a $5,33 \mathrm{mmHg}$ das mulheres saudáveis ( $p$ $=0,0009$ ) (recuperação mais lenta). Pode-se inferir que o HS é capaz de causar disfunções cárdio-pulmonares, com maior sensibilidade para os parâmetros previamente citados. (Arq Bras Endocrinol Metab 2007;51/9:1485-1492)
\end{abstract}

Descritores: Hipotireoidismo; Avaliação da capacidade funcional; Pressão arterial; Consumo de oxigênio; Limiar anaeróbico

\section{ABSTRACT}

\section{Impact of Subclinical Hypothyroidism in Cardiopulmonary} Response During Effort and its Recovery.

In order to identify the characteristics of subclinical hypothyroidism (SH) during physical stress and its recovery, $15 \mathrm{SH}$ patients and 16 healthy women were compared by a treadmill cardiopulmonary test. Means of variables were analyzed by the Mann-Whitney $U$ test. Patients obtained lower values for peak expired fraction of $\mathrm{O}_{2}(14.90 \pm 1.05 \times 16 \pm 1.14 \% ; \mathrm{p}=$ $0.014)$; systolic blood pressure variation $(34.33 \pm 17.92 \times 52.50 \pm 17.22 ; p=$ $0.009)$; exercise duration ( $8.83 \pm 2.91 \times 14.5 \pm 5.63 \mathrm{~min} ; \mathrm{p}=0.0005)$, maximal test load $(11.6 \pm 4.22 \times 18.94 \pm 5.45 \% ; p=0.0004)$, as well as tendencies in gas exchange ratio and peak heart rate. Between the first and the third recovery minutes, there was a reduction of only $0.71 \mathrm{mmHg}$ in the diastolic blood pressure, whereas there was a $5.33-\mathrm{mmHg}$ reduction to control group ( $p=0.0009$ ) (slower recovery of patients). It is presumable that $\mathrm{SH}$ may cause cardiopulmonary dysfunctions, with higher sensibility to the parameters previously cited. (Arq Bras Endocrinol Metab 2007;51/9:1485-1492)

Keywords: Hypothyroidism; Work capacity evaluation; Blood pressure; Oxygen consumption; Anaerobic threshold

\section{artigo original}

\author{
MÍRIAM R.M. MAINENTI \\ PATRÍCIA F.S. TEIXEIRA \\ FÁtIMA P. OLIVEIRA \\ MÁrIo VAISMAN
}

Programa de Pós-Graduação em Endocrinologia / Serviço de Endocrinologia - HUCFF, e Escola de Educação Física e Desportos (Laboratório de Fisiologia do Exercício) Universidade Federal do Rio de Janeiro, RJ.

Recebido em 21/1 1/06

Aceito em 06/08/07 
$\mathrm{O}$ HIPOTIREOIDISMO SUBCLÍNICO (HS) É caracterizado por um valor sérico aumentado do hormônio tireoestimulante (TSH) e concentrações normais de tiroxina livre (T4L) (1). A sua prevalência varia em função da localidade, do sexo e da faixa etária estudada. Estudos realizados na Arábia Saudita revelaram que o HS apresenta uma prevalência de $35 \%$ em mulheres acima de 50 anos (2), enquanto que nos Estados Unidos as estatísticas apontam para 4,3\% de prevalência em homens e mulheres acima dos 12 anos (3). Recentemente, verificou-se uma prevalência de $3,0 \%$ de tal entidade em funcionários da Universidade Federal do Rio de Janeiro, Brasil (4). Resultados ainda não publicados de um estudo epidemiológico do nosso grupo mostraram HS em 7,27\% das participantes (amostra de 1.292 mulheres acima de 35 anos).

Diversos estudos analisam sinais e sintomas possivelmente associados ao HS, mas são raras as investigações sobre a capacidade funcional de pacientes portadores de HS. Entende-se como capacidade funcional a resposta integrada dos sistemas cárdio-pulmonar, circulatório e metabólico ao esforço. Ela pode ser medida pelo teste ergoespirométrico, que é caracterizado pela medida direta das trocas gasosas. A ergoespirometria é um teste de fácil aplicação que permite a medida da resposta ao esforço da ventilação (volume inspirado e expirado) e das frações gasosas presentes nesses volumes (5). A partir dessas medidas obtêm-se parâmetros como o consumo máximo de oxigênio ( $\left.\mathrm{V}_{\mathrm{O} 2 \text { máx }}, 1 . \mathrm{min}^{-1}\right)$, produção de gás carbônico $\left(\mathrm{V}_{\mathrm{CO} 2 \mathrm{máx}}, 1 . \mathrm{min}^{-1}\right)$, Limiar anaeróbio (LA, $\left.1 . \mathrm{min}^{-1}\right)$, tempo de exercício e resposta hemodinâmica tanto no esforço como em sua recuperação.

Estudos anteriores demonstraram que pacientes portadores de HS apresentam comportamentos indicativos de alteração do sistema cárdio-pulmonar, como também altos índices de relato de fadiga precoce (2); baixo $\mathrm{V}_{\mathrm{O} 2 \max }(6)$ e LA precoce (7). Entretanto, a lite- ratura ainda não é consistente em relação aos parâmetros afetados pela doença que se manifestam durante o esforço e na sua recuperação.

$\mathrm{O}$ presente estudo tem como objetivo prover à comunidade científica dados sobre a capacidade funcional de pacientes com hipotireoidismo subclínico. Pretende-se identificar as manifestações clínicas que ocorrerem durante o esforço físico e na recuperação.

\section{MATERIAIS E MÉTODOS}

\section{Pacientes}

O estudo caracteriza-se por uma análise transversal de mulheres entre 30 e 60 anos pertencentes a dois grupos de estudo, que foram comparados. O primeiro composto por portadoras de HS e o segundo por mulheres sem comprometimento tireoidiano (tabela 1 ). Os grupos foram comparáveis quanto às variáveis tabagismo e menopausa. As pacientes foram recrutadas a partir de um estudo populacional e encaminhadas ao ambulatório de Endocrinologia do Hospital Universitário Clementino Fraga Filho, da Universidade Federal do Rio de Janeiro (HUCFF/UFRJ), para avaliação da possibilidade de inserção na pesquisa considerando os critérios de inclusão e exclusão.

Os critérios de inclusão para HS foram: I) duas dosagens (com intervalo mínimo de 4 semanas) de TSH acima do limite superior de normalidade adotado $(4,0 \mathrm{mU} / \mathrm{l})$; II) nível de T4 livre dentro da faixa da normalidade $(0,8$ a 1,9 $\mathrm{ng} / \mathrm{dl})$.

Os critérios de inclusão no grupo controle foram: I) dosagem de TSH $(0,4-4,0 \mathrm{mU} / \mathrm{l})$ e T4L na faixa da normalidade; II) pesquisa negativa de ATPO; III) ausência de história de doença tireoidiana e IV) exame físico da glândula tireoidiana nos limites da normalidade.

Os critérios de exclusão foram: I) uso de drogas ou substâncias que interferem na função tireoidiana, a ponto de alterar dosagens séricas (lítio, amiodarona, glicocorticóides, entre outras); II) presença de doenças, mesmo que estáveis - que alterem os níveis circulantes dos hormônios

Tabela 1. Características principais dos grupos paciente e controle.

\begin{tabular}{|c|c|c|c|}
\hline & Paciente & Controle & Valor $\mathbf{p}^{\Phi}$ \\
\hline Idade (anos) & $42,2 \pm 7,51$ & $38,69 \pm 7,62$ & 0,14 \\
\hline Massa Corporal Total (kg) & $73,11 \pm 16,83$ & $64,31 \pm 8,66$ & 0,25 \\
\hline Estatura (m) & $1,59 \pm 0,08$ & $1,60 \pm 0,07$ & 0,86 \\
\hline T4L (ng.dl-1) & $1,08 \pm 0,19$ & $1,44 \pm 0,87$ & 0,13 \\
\hline TSH $\left(m U . I^{-1}\right)$ & $7,35 \pm 3,78$ & $1,14 \pm 0,19$ & $0,000016^{*}$ \\
\hline Tabagismo (Sim/Não/Ex-fumante) & $(2 / 9 / 4)$ & $(4 / 10 / 2)$ & 1,00 \\
\hline Menopausa (Sim/Não) & $(3 / 12)$ & $(3 / 13)$ & 1,00 \\
\hline Reposição Hormonal (n) & 1 & 1 & \\
\hline
\end{tabular}


tireoidianos (síndrome nefrótica, insuficiência renal, insuficiência hepática, AIDS); III) uso de levotiroxina; IV) doença infecciosa em evolução; V) arritmias potencialmente graves; VI) infarto e/ou angina nos últimos 3 meses; VII) uso de medicamentos que interferem na freqüência cardíaca e/ou pressão arterial; VIII) dores ou outros problemas físicos que impeçam a caminhada; IX) apresentação de pressão arterial sistólica (PAS) maior do que $200 \mathrm{mmHg}$ e/ou a pressão arterial diastólica (PAD) maior do que 110 $\mathrm{mmHg}$ no momento do teste e X) participação em programa de atividade física regular.

O presente estudo foi aprovado pelo comitê de ética do HUCFF/Faculdade de Medicina. As pacientes assinaram o termo de consentimento livre e esclarecido na primeira consulta no serviço de endocrinologia do HUCFF/UFRJ, e em seguida foram encaminhadas para o Laboratório de Fisiologia do Exercício da Escola de Educação Física e Desportos da Universidade (Labofise/EEFD/UFRJ), onde foi realizado o teste ergoespirométrico.

\section{Ergoespirometria}

\section{Recomendações prévias}

No momento da marcação do teste, foi requerido das participantes que não realizassem atividade física extenuante no dia anterior ao teste, não ingerissem bebidas alcoólicas ou cafeína no dia ou na noite anterior, e não fumassem nas últimas quatro horas antes do exame.

\section{Preparação pré-teste}

Antes do início do testes, dez eletrodos prata/cloreto de prata $(\mathrm{Ag} / \mathrm{AgCl})$ descartáveis eram acoplados à pele devidamente higienizada, para obtenção do eletrocardiograma (ECG) padrão de 12 derivações $(6$ periféricas e 6 precordiais). A próxima etapa consistia em medir a pressão arterial (PA) em repouso de pé (PA pré-teste) e colocar na participante a máscara para ventilação, usando um clipe para privar a passagem de ar pelas narinas.

$\mathrm{O}$ teste ergoespirométrico foi realizado em uma esteira ergométrica da marca Ecafix EG 700.2, utilizando o protocolo de Balke modificado, que consiste no aumento gradativo de $3 \%$ da inclinação da esteira a cada 2 minutos, com velocidade constante e igual a $4,8 \mathrm{~km} / \mathrm{h}$. A PA foi medida a cada três minutos do período de exercício e na recuperação ( lo e $^{\circ}$ o minutos), utilizando-se de um tensiômetro de coluna de mercúrio da marca Narcosul, modelo 1400-C.

Todos os procedimentos foram explicados às participantes, incluindo os critérios de interrupção do teste, dentre as quais: início de angina, queda significativa ou ausência de elevação na pressão sistólica com um aumento na intensidade do exercício, pressão sistólica maior que 220 $\mathrm{mmHg}$ ou pressão diastólica maior que $115 \mathrm{mmHg}$, tonteira, confusão, palidez, ausência do aumento na freqüência cardíaca com uma maior intensidade do exercício por um período prolongado, alterações no sinal do eletrocardiograma, manifestações físicas ou verbais de fadiga extrema. A interrupção se dava no momento em que se tinha o aparecimento de um desses sintomas ou quando ela solicitasse. Durante todo o teste, a paciente era questionada a respeito da percepção do esforço realizado, através da escala de Borg (variando de 6 a 20 - extremamente leve a extremamente intenso). Para controle dos sintomas limitantes foram observadas as diretrizes do American College of Sports Medicine (8).

A ergoespirometria é uma técnica utilizada para avaliar a capacidade cárdio-pulmonar de um indivíduo pela análise direta dos gases respirados. A técnica reproduz uma medida direta da capacidade funcional do indivíduo, evitando erros decorrentes de fórmulas da ergometria simples e de outros testes de campo que estimam essa capacidade a partir da duração do teste, da carga máxima ou da velocidade final alcançada. Além disso, é uma técnica não-invasiva, o que aumenta a acessibilidade da sua realização (9).

A partir das frações expiradas de $\mathrm{O}_{2}$ e $\mathrm{CO}_{2}\left(\mathrm{FE}_{\mathrm{O} 2}\right.$, $\left.\mathrm{FE}_{\mathrm{CO} 2}\right)$ e da ventilação pulmonar $\left(\mathrm{V}_{\mathrm{E}}=\right.$ Volume expirado $\mathrm{X}$ Freqüência respiratória) são obtidos importantes parâmetros para a interpretação clínica dos pacientes.

Já está consolidada na literatura a técnica de identificação do LA através da técnica ventilatória (9). Os critérios adotados para a sua caracterização foram: perda de linearidade da $\mathrm{V}_{\mathrm{E}}$; tendência de ascensão abrupta de $\mathrm{R}$ após alcançar o valor um; aumento sistemático da $\mathrm{V}_{\mathrm{E}} / \mathrm{V}_{\mathrm{O} 2}$, sem um aumento correspondente na $\mathrm{V}_{\mathrm{E}} / \mathrm{V}_{\mathrm{CO} 2}$ (9-11). Identificado o momento no teste de esforço em que o LA ocorreu, este é expresso pelo valor de $\mathrm{V}_{\mathrm{O} 2}$ em que o paciente se encontra.

Os sinais eletrocardiográficos (ECG) foram gravados através do sistema Ecafix Cardio Perfect ECG, observados e armazenados pelo programa Medical Diagnostic Workstation - Cardio Control 2000/2001. A freqüência cardíaca (FC), bem como todo o traçado do ECG, era apresentados em tempo real para monitoramento da avaliada. O sistema de aquisição de sinais ventilatórios é composto por um pneumotacógrafo (MEDICAL GRAPHICS, fluxo médio) que, acoplado a um transdutor de pressão diferencial, faz a leitura do fluxo de ar expirado. A curva de vazão obtida é integrada para se obter os volumes inspirados e expirados. Sincronizado com as medidas de volume, um analisador de gases (MEDICAL GRAPHICS - modelo VO2000) capta amostras a cada ciclo respiratório e fornece as frações gasosas da inspiração e expiração. A análise dos gases foi feita ciclo a ciclo, amostrando valores como uma média de cada três ciclos.

\section{Parâmetros}

\section{Cárdio-pulmonares}

Ventilação minuto de pico (no fim do exercício) ( $\mathrm{V}_{\text {Epico, }}$ l.min ${ }^{-1}$, STPD); consumo de oxigênio de pico $\left(\mathrm{V}_{\mathrm{O} \text { pico }}\right.$, $\mathrm{STPD})$ e produção de gás carbônico de pico $\left(\mathrm{V}_{\mathrm{CO} 2 \text { pico }}\right.$ STPD), tanto absoluto (1. $\left.\mathrm{min}^{-1}\right)$ como relativo $\left(\mathrm{ml} . \mathrm{kg} \cdot \mathrm{min}^{-1}\right)$; 
equivalente ventilatório de oxigênio de pico $\left(\mathrm{V}_{\mathrm{E}} / \mathrm{V}_{\mathrm{O} \text { pico }}\right)$; equivalente ventilatório de gás carbônico de pico $\left(\mathrm{V}_{\text {Epico }} / \mathrm{V}_{\mathrm{CO} 2 \text { pico }}\right)$; fração expirada de oxigênio de pico $\left(\mathrm{FE}_{\mathrm{O} 2 \mathrm{pico}}\right)$; fração expirada de gás carbônico de pico $\left(\mathrm{FE}_{\mathrm{CO2pico}}\right)$; razão de trocas gasosas de pico $\left(R_{\text {pico }}=V_{\text {CO2pico }}\right.$ $\left.\div V_{O 2 p i c o}\right)$; pulso de oxigênio de pico $\left(P O_{\text {pico }}=V_{O 2} \div F C\right.$; l.min.bat $\left.{ }^{-1}\right)$; LA $\left(1 . \mathrm{min}^{-1}\right)$.

\section{Hemodinâmicos}

1.Pré-teste: pressão arterial sistólica (PAS) e diastólica (PAD) e freqüência cardíaca $\left(\mathrm{FC}\right.$, bat. $\left.\mathrm{min}^{-1}\right)$;

2.No fim do exercício (ou pico): $\mathrm{PAS}_{\text {pico, }} \mathrm{PAD}_{\text {pico }}$ e $\mathrm{FC}_{\text {pico }}$. Foi calculada a diferença entre os valores alcançados no pico do exercício e os valores do pré-teste para PAS e PAD. Duplo produto de pico $\left(\mathrm{DP}_{\text {pico }}\right)$, que expressa a relação entre a FC e a PAS $(D P=F C \times P A S)$, e o $P_{2 \text { pico, }}$ que expressa a quantidade de $\mathrm{O}_{2}$ enviado por batimento cardíaco, também foram calculados;

3.Recuperação: Medidas de PA no primeiro (PAS Recl e PAD Recl) e no terceiro minuto (PAS Rec3 e PAD Rec3) após a interrupção do exercício. Foi calculada a diferença entre os valores encontrados no pico e em todos os momentos monitorados da recuperação, de modo a se obter a diferença de retorno às condições basais dos grupos.

\section{Funcionais}

Duração do teste, carga de trabalho de pico, percepção de esforço de pico (Borg pico).

\section{Análise estatística}

A comparação das médias das variáveis dos resultados obtidos entre os grupos foi realizada pelo teste estatístico de Mann-Whitney. O teste exato de Fisher foi empregado para comparar a proporção de pacientes quanto às variáveis menopausa e tabagismo. Para essas análises foi utilizado o programa Statistica versão 5.5 (StatSoft), considerando o nível de significância de 0,05 .

\section{RESULTADOS}

Foram incluídas 15 pacientes portadoras de HS e 16 mulheres sem comprometimento tireoidiano, cujas características estão expostas na tabela 1 .

\section{Parâmetros cárdio-pulmonares}

$\mathrm{O} \mathrm{V}_{\text {O2pico }}$ o $\mathrm{V}_{\mathrm{CO} 2 \text { pico }}$ (absoluto e relativo) e a $\mathrm{V}_{\text {Epico }}$ não apresentaram diferenças entre os grupos. Entretanto, no pico do exercício, a razão de trocas gasosas; o pulso de oxigênio; os equivalentes ventilatórios e as frações expiradas de oxigênio e gás carbônico demonstraram-se estatisticamente diferentes entre os dois grupos (tabela 2).

No HS foram encontrados menores valores para a $\mathrm{FE}_{\text {O2pico }}(\mathrm{p}=0,01)$, enquanto que valores de $\mathrm{FE}_{\mathrm{CO} 2 \text { pico }}(\mathrm{p}=0,01)$ foram maiores que no grupo de controle (figura 1 ).

O LA foi alcançado em apenas $13 \%$ das mulheres com HS e $50 \%$ das controles. Tendo em vista essa condição metabólica diferenciada entre os grupos, todos os parâmetros foram analisados apenas no momento de esforço máximo, e não no limiar.

\section{Parâmetros hemodinâmicos}

$\mathrm{Na}$ análise dos parâmetros hemodinâmicos, as diferenças estatisticamente significativas foram encontra-

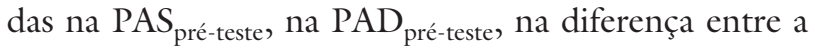
$\mathrm{PAS}_{\text {pico }}$ e a PAS pré-teste (PAS P-PT) e na recuperação da PAD entre o primeiro e o terceiro minutos após o esforço (PAD DIF 1-3). Tendências não comprovadas estatisticamente foram observadas na PAS do primeiro minuto de recuperação (PAS Rec 1), na alteração da PAS entre o primeiro e o terceiro minutos após o esforço (PAS DIF 1-3) e na $\mathrm{FC}_{\text {pico }}$ (tabela 3).

Tabela 2. Resultados dos parâmetros cárdio-pulmonares.

\begin{tabular}{|c|c|c|c|}
\hline & Pacientes & Controle & Valor $\mathbf{p}^{\Phi}$ \\
\hline $\mathrm{V}_{\text {Epico }}\left(\mathrm{I} \cdot \mathrm{min}^{-1}\right)$ & $40,06 \pm 16,09$ & $42,57 \pm 12,12$ & 0,338 \\
\hline $\mathrm{V}_{\mathrm{O} 2 \mathrm{pico}}\left(\mathrm{I} \cdot \mathrm{min}^{-1}\right)$ & $2,33 \pm 0,93$ & $1,95 \pm 0,43$ & 0,379 \\
\hline $\mathrm{V}_{\text {O2pico }}\left(\mathrm{ml} \cdot \mathrm{kg} \cdot \mathrm{min}^{-1}\right)$ & $31,75 \pm 9,37$ & $30,26 \pm 4,82$ & 0,520 \\
\hline $\mathrm{V}_{\text {CO2pico }}\left(1 . \mathrm{min}^{-1}\right)$ & $2,02 \pm 0,9$ & $1,87 \pm 0,53$ & 0,870 \\
\hline$V_{\text {CO2pico }}\left(\mathrm{ml} \cdot \mathrm{kg} \cdot \mathrm{min}^{-1}\right)$ & $27,07 \pm 8,57$ & $29,13 \pm 7,18$ & 0,861 \\
\hline $\mathrm{FE}_{\mathrm{O} 2 \mathrm{pico}}(\%)$ & $14,90 \pm 1,05$ & $16,00 \pm 1,14$ & $0,014^{*}$ \\
\hline $\mathrm{FE}_{\mathrm{CO} 2 \text { pico }}(\%)$ & $4,98 \pm 0,84$ & $4,44 \pm 0,48$ & $0,019 *$ \\
\hline $\mathrm{R}_{\text {pico }}$ & $0,85 \pm 0,12$ & $0,96 \pm 0,17$ & $0,054^{\Delta}$ \\
\hline $\mathrm{V}_{\mathrm{O} 2} / \mathrm{FC}_{\text {pico }}\left(\right.$ I.bat $\left.^{-1}\right)$ & $0,015 \pm 0,005$ & $0,012 \pm 0,003$ & $0,078^{\Delta}$ \\
\hline
\end{tabular}



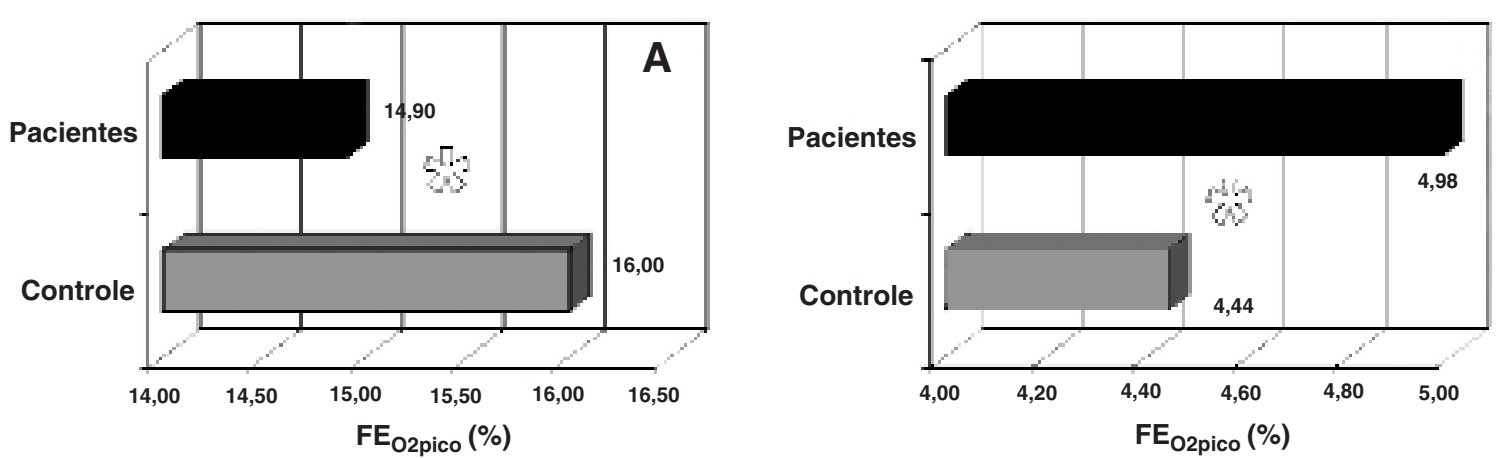

B

Figura 1. A) Fração Expirada de $\mathrm{O}_{2}\left(\mathrm{FE}_{\mathrm{O} 2 \text { pico }}\right)$; e $\left.\mathrm{B}\right)$ Fração Expirada de $\mathrm{CO}_{2}\left(\mathrm{FE}_{\mathrm{CO} \text { pico }}\right)$ em controles e pacientes. Diferença encontrada por meio do Mann-Whitney U Test ( $p=0,014$; e 0,019, respectivamente).

Tabela 3. Resultados dos parâmetros hemodinâmicos.

\begin{tabular}{|c|c|c|c|}
\hline & Pacientes & Controle & Valor $\mathbf{p}^{\Phi}$ \\
\hline $\mathrm{PAS}_{\text {pré-teste }}$ & $120,66 \pm 11,93$ & $112,18 \pm 7,74$ & $0,017 *$ \\
\hline PAS $_{\text {pico }}$ & $155,00 \pm 23,98$ & $164,68 \pm 20,37$ & 0,216 \\
\hline PAS Rec 1 & $144,28 \pm 19,10$ & $155,93 \pm 20,43$ & $0,077^{\triangle}$ \\
\hline PAS Rec 3 & $133,66 \pm 14,33$ & $136,33 \pm 21,59$ & 0,775 \\
\hline $\mathrm{PAD}_{\text {pré-teste }}$ & $80,33 \pm 6,67$ & $73,75 \pm 6,95$ & $0,014^{*}$ \\
\hline $\mathrm{PAD}_{\text {pico }}$ & $88,00 \pm 7,51$ & $88,75 \pm 7,19$ & 0,922 \\
\hline PAD Rec 1 & $84,28 \pm 8,96$ & $84,68 \pm 11,03$ & 0,951 \\
\hline PAD Rec 3 & $84,00 \pm 7,84$ & $80,33 \pm 7,9$ & 0,217 \\
\hline PAS DIF 1 & $9,64 \pm 13,37$ & $8,75 \pm 11,62$ & 0,854 \\
\hline PAS DIF 3 & $21,33 \pm 18,07$ & $27,33 \pm 14,86$ & 0,137 \\
\hline PAS DIF 1-3 & $11,43 \pm 10,64$ & $20,33 \pm 14,07$ & $0,051 \Delta$ \\
\hline PAS P-PT & $34,33 \pm 17,92$ & $52,50 \pm 17,22$ & $0,009 *$ \\
\hline PAD DIF 1 & $4,29 \pm 5,14$ & $4,06 \pm 7,12$ & 0,448 \\
\hline PAD DIF 3 & $10,67 \pm 13,48$ & $8,33 \pm 5,23$ & 0,713 \\
\hline PAD DIF 1-3 & $0,71 \pm 2,67$ & $5,33 \pm 4,42$ & $0,009 *$ \\
\hline PAD P-PT & $7,67 \pm 6,78$ & $15,00 \pm 8,56$ & 0,017 \\
\hline $\mathrm{FC}_{\text {pré-teste }}$ & $82,93 \pm 12,96$ & $80,25 \pm 9,7$ & 0,520 \\
\hline $\mathrm{FC}_{\text {pico }}$ & $154,13 \pm 19,96$ & $167,81 \pm 17,8$ & $0,060^{\Delta}$ \\
\hline $\mathrm{DP}_{\text {pico }}$ & $24061,67 \pm 5561,08$ & $27713,13 \pm 4777,03$ & 0,119 \\
\hline
\end{tabular}

Os valores estão dispostos como média \pm desvio-padrão.

$\Phi$ Calculado através do Mann-Whitney U Test

* Valores significativos para $p<0,05$

$\Delta$ Valores de p limítrofe, tendência não estatística

Comparando o comportamento da PAS entre pacientes e controles, a PAS P-PT, que é a diferença entre a PAS de pico e a PAS no pré-teste, foi menor no HS (figura 2).

A recuperação da pressão arterial diastólica (PAD) no HS apresentou-se mais lenta. No terceiro minuto de recuperação houve um decréscimo (em relação ao primeiro minuto de recuperação) em $0,71 \mathrm{mmHg}$ na $\mathrm{PAD}$, enquanto que no grupo controle diminuiu, em média, $5,33 \mathrm{mmHg}(\mathrm{p}=0,0009)$ (figura 3 ).

Os valores de freqüência cardíaca no final do teste das pacientes tenderam a ser menores do que os das mulheres controles (tabela 3). Entretanto, ao comparar a FC em uma mesma intensidade de trabalho (9\% inclinação), o contrário foi encontrado: as portadoras de HS $(139,29 \pm 16,98 \mathrm{bpm})$ apresentaram maiores valores do que as mulheres controle $(131,07 \pm 14,55 \mathrm{bpm})$, sem comprovação estatística $(\mathrm{p}=0,112)$ (figura 4$)$.

\section{Parâmetros funcionais}

As pacientes obtiveram valores menores na duração do exercício $(8,83 \pm 2,91 \mathrm{~min} \times 14,5 \pm 5,63 \mathrm{~min} ; \mathrm{p}=$ $0,0005)$ e na carga máxima de teste $(11,6 \pm 4,22 \%$ inclinação x 18,94 $\pm 5,45 \%$ inclinação, $\mathrm{p}=0,0004$ ) 


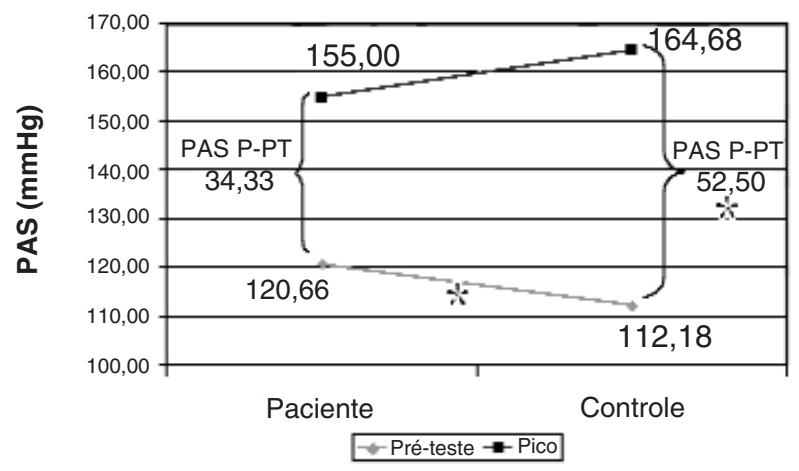

Figura 2. Valores médios da PAS das pacientes e das mulheres controle no pré-teste (PAS pré-teste), no pico $\left(\mathrm{PAS}_{\text {pico }}\right)$ e a diferença entre eles (PAS P-PT). Os asteriscos $(*)$ marcam a presença de diferença comprovada pelo MannWhitney $U$ Test $(p=0,05)$.

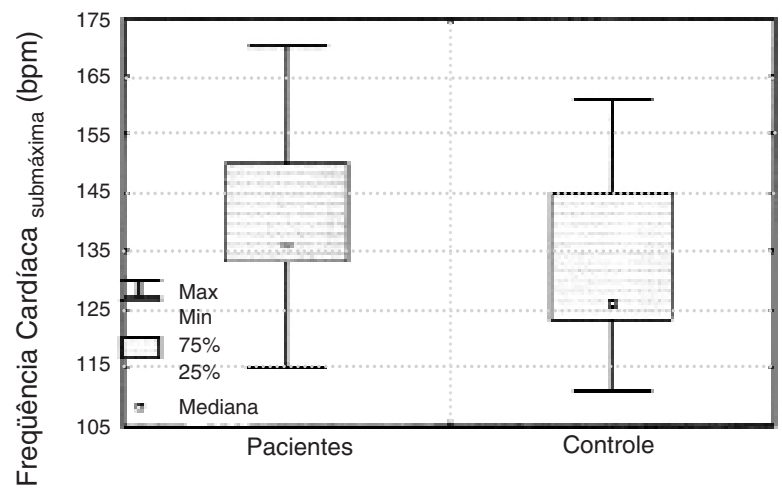

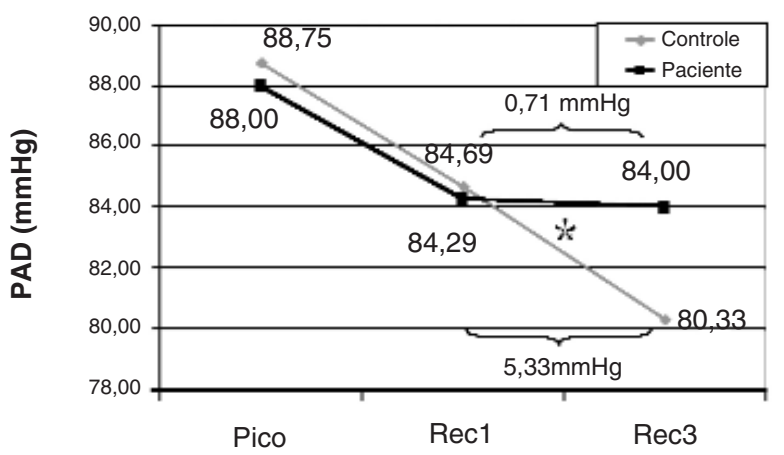

Figura 3. Valores médios da PAD das pacientes e das mulheres controle no pico, no primeiro e no terceiro minuto de recuperação (Rec1 e Rec3). O asterisco (*) marca a presença de diferença entre as variações de pressão de Rec 1 para Rec 3 (PAD Rec 1-3) dos dois grupos, pelo MannWhitney $U$ Test $(p=0,05)$.

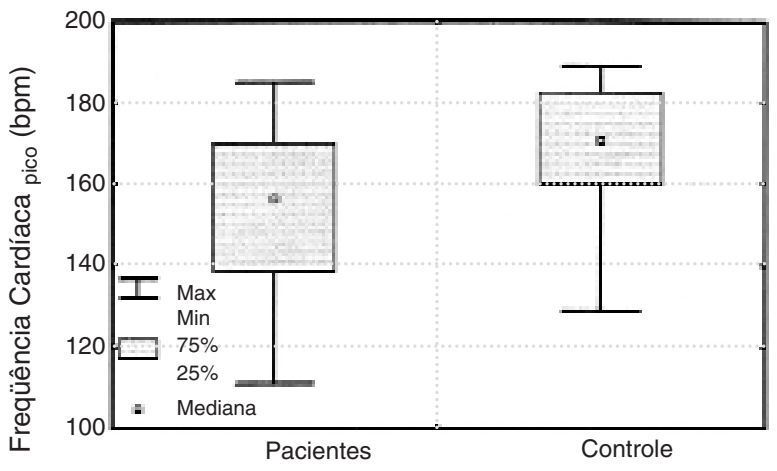

Figura 4. Box plot da freqüência cardíaca submáxima e de pico (bpm) para os grupos paciente e controle. São demonstrados os valores mínimo, primeiro quartil $(25 \%)$, mediana, terceiro quartil $(75 \%)$ e máximo para cada grupo. Intensidade submáxima $=9 \%$ inclinação; intensidade de pico = imediatamente antes de terminar o teste.

(figura 5), ambos os grupos com uma percepção média do esforço no pico do exercício como "muito intenso" (valor 17 da escala de percepção de esforço de Borg).

\section{DISCUSSÃo}

\section{Parâmetros cárdio-pulmonares}

A medida do $\mathrm{V}_{\mathrm{O} 2}$ reflete as necessidades energéticas aeróbias do organismo, permitindo a obtenção de informações precisas da capacidade de transporte e utilização do oxigênio, isto é, da capacidade funcional dos pulmões e do sistema cardiovascular, muscular e mitocondrial, combinados. O momento do exercício em que a capacidade de fornecimento de oxigênio aos músculos em atividade é saturada é denominado de consumo máximo de oxigênio $\left(\mathrm{V}_{\mathrm{O} 2 \text { máx }}\right)$. Pessoas não treinadas encerram o exercício antes de atingir essa situação, assim, o maior valor de $\mathrm{V}_{\mathrm{O} 2}$ alcançado é registrado e denominado $\mathrm{V}_{\mathrm{O} \text { pico }}(9,12)$.

Os valores do $\mathrm{V}_{\text {O2pico }}$ (relativo ou absoluto) de pacientes e controles não diferiram no presente experimento, o que expressa a ausência de impacto detectável do HS no sistema cárdio-respiratório através dessa variável para o grupo estudado. Esse resultado difere do encontrado por um grupo de pesquisadores da Itália (6), que obteve $\mathrm{V}_{\text {O2pico }}$ reduzido no grupo de pacientes com hipotireoidismo subclínico.

A avaliação cárdio-pulmonar revelou, ainda, que as pacientes apresentaram uma menor resistência à 

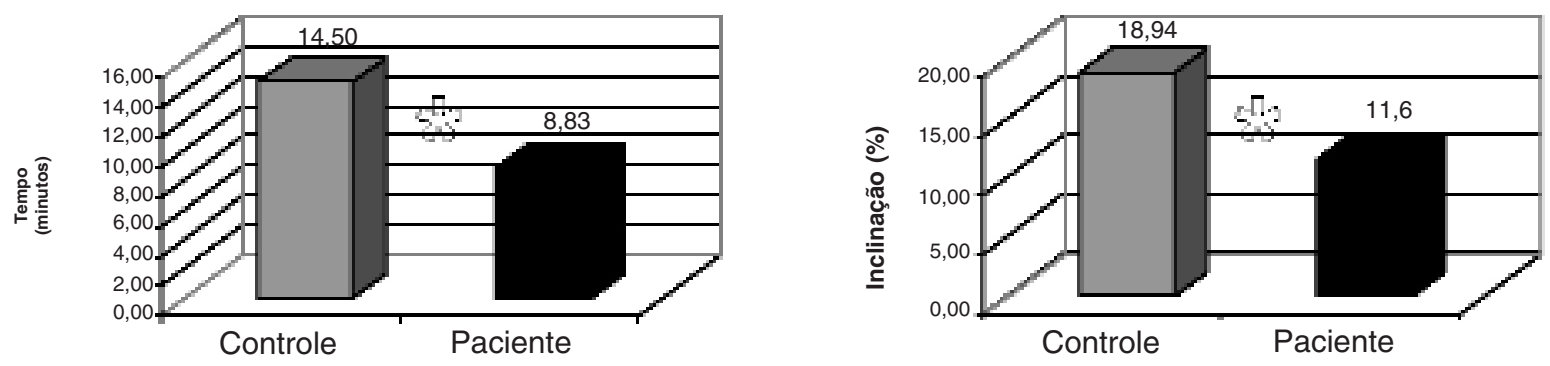

Figura 5. Duração e carga máxima do teste nos grupos controle e paciente. Diferença encontrada por meio do Mann-Whitney U Test ( $p=0,0005$ e 0,0004).

acidose metabólica celular proveniente do aumento da contribuição do metabolismo anaeróbio (glicólise) durante o esforço. Essa resposta foi evidenciada pela instalação da fadiga antes de alcançarem o LA. Essa fadiga precoce detectada nas pacientes pode ser devida a uma pobre resposta ventilatória à acidose metabólica da parte da paciente (9), ou mesmo à menor capacidade de metabolizar o lactato produzido e, ainda, à insegurança de se conceber como "paciente", interrompendo o exercício em condição submáxima [mesmo não encontrando diferença estatística entre os grupos para o valor de percepção de esforço no final do teste, o valor modal da escala de Borg de pico nas pacientes é menor (15) em comparação com as mulheres controle (17)].

Com o incremento da intensidade de um exercício, o organismo passa a utilizar mais substrato glicídico para geração de energia (13). O maior valor de $\mathrm{FE}_{\mathrm{CO} 2}$ alcançado pelas pacientes (figura $1 \mathrm{~B}$, tabela 2 ) representa uma intensidade relativa maior para as pacientes e/ou uma tolerância menor a uma dada intensidade. Aumentando a quantidade de $\mathrm{CO}_{2}$ produzido internamente, os quimio-receptores são estimulados e esse processo gera uma resposta reflexa de aumento da ventilação pulmonar (13), o que aconteceu em ambos os grupos (controle e paciente) sem diferença no momento de pico (tabela 2).

\section{Parâmetros hemodinâmicos}

De acordo com artigos recentes de revisão $(14,15)$, há uma diminuição na contratilidade do miocárdio em pacientes acometidos pelo HS. Entretanto, nas pacientes estudadas na presente análise, essa disfunção sistólica não acometeu valores aferidos em repouso, sendo a PAS pré-teste média de $120,66 \mathrm{mmHg}$, ou seja, encontra-se dentro da faixa de normalidade.

Em esforço, há algumas mudanças no comportamento da pressão arterial das pacientes. Um achado interessante é a menor variação pico-repouso. A figura 2 apresenta valores pré-teste, no pico e essa variação para os grupos analisados. Mesmo iniciando com uma PAS maior, as pacientes não conseguiram evoluir tanto quanto as mulheres do grupo controle, demonstrando que a diminuição da contratilidade do miocárdio, citada previamente, deixa de ser silenciosa em exercício.

Já é conhecida a presença de uma disfunção diastólica, tanto para o hipotireoidismo manifesto quanto para o subclínico $(16,17)$. A diferença encontrada na PAD pré-teste entre os grupos se deu, provavelmente, pelo aumento da resistência vascular periférica inerente aos pacientes com hipotireoidismo (1719). Além de os valores pré-teste estarem alterados, outro resultado que retrata o comprometimento da função diastólica é uma recuperação menos eficiente da PAD nas pacientes, apresentada por uma recuperação mais lenta entre o primeiro e o terceiro minutos (PAD DIF 1-) (figura 3). Os valores de PAD DIF 1 e PAD DIF 3 também foram menores nesse grupo (mesmo sem significância estatística), expressando uma pior capacidade de recuperação da PAD nos primeiros minutos após um esforço de alta intensidade.

As alterações observadas na pressão arterial não foram acompanhadas por modificações na freqüência cardíaca de repouso. Esse resultado confirma os achados de outros estudos $(16,20)$, que encontraram freqüência cardíaca de repouso normal em todos os seus pacientes. Em relação à $\mathrm{FC}_{\text {pico, }}$ os resultados mostram uma tendência de menores valores para as pacientes $(\mathrm{p}=0,06)$, que poderia (ou não) ser mais do que uma tendência com um maior tamanho amostral (figura 4). Mesmo com essa tendência para ser menor no momento do pico, em cargas submáximas o contrário acontece (demonstrado na comparação para uma mesma intensidade absoluta — 9\% de inclinação). 
Mesmo não tendo sido obtida significância estatística, esse comportamento pode demonstrar um trabalho menos eficiente do coração no grupo dessas pacientes.

$\mathrm{O}$ menor valor de $\mathrm{FC}_{\text {pico }}$ nas pacientes explica a diferença (não estatística) no parâmetro pulso de oxigênio, maior para esse grupo.

\section{Parâmetros funcionais}

Os dados do presente estudo sugerem menor duração e da carga de pico para as pacientes (figura 5). Esses resultados confirmam a deficiência em conseguir suportar cargas mais intensas no hipotireoidismo subclínico. Em média, as mulheres do grupo controle conseguiram alcançar uma intensidade na esteira de 19\% de inclinação, enquanto que as pacientes atingiram $11,5 \%$. Mesmo diferentes em termos absolutos, a percepção na escala de Borg (2l) das duas intensidades (19 e 11,5\%) foi de "muito difícil" para ambos os grupos. A duração do teste e a carga de pico são parâmetros pouco explorados na literatura, entretanto devem receber atenção especial por serem altamente relacionados às atividades cotidianas dessas pacientes.

Os resultados obtidos nos parâmetros cárdiopulmonares $\left(\mathrm{V}_{\mathrm{O} \text { pico }}\right.$ semelhante para ambos os grupos; menores valores para $\mathrm{FE}_{\mathrm{O} 2} \mathrm{e} \mathrm{R}$; caracterização do limiar anaeróbico em apenas $13 \%$ pacientes) somados aos resultados hemodinâmicos (menor $\mathrm{PAS}_{\text {pico }}$, menor $\mathrm{FC}_{\text {pico }}$ ) e funcionais (menor duração e carga máxima de teste) parecem indicar uma avaliação submáxima nos testes realizados pelas pacientes. Além disso, o comprometimento da recuperação da PAD e os resultados funcionais demonstram que as pacientes acometidas pelo hipotireoidismo subclínico possuem uma baixa tolerância a esforços mais intensos e um pior condicionamento físico em comparação com mulheres saudáveis.

\section{AGRADECIMENTOS}

Os autores agradecem ao laboratório Abbott pelo auxílio financeiro, e aos estudantes de educação física Adriana Macedo e Maicon Maia pela assistência operacional, na realização dos testes ergoespirométricos.

\section{REFERÊNCIAS}

1. Surks M, Ortiz E, Daniels G, Sawin C, Col N, Cobin R, et al. Subclinical thyroid disease: scientific review and guidelines for diagnosis and management. JAMA 2004;291/2:228-38.

2. Akbar D, Ahmed M, Hijazi N. Subclinical hypothyroidism in elderly women attending an outpatient clinic. Med Sci Monit 2004;10/5:229-32.
3. Hollowell JG, Staehling NW, Flanders WD, Hannon WH, Gunter EW, Spencer CA, et al. Serum TSH, $\mathrm{T}_{4}$, and thyroid antibodies in the United States population (1988 to 1994): National Health and Nutrition examination Survey (NHANES III). J Clin Endocrinol Metab 2003;87/2:489-99.

4. REIS FAA. Prevalência de alterações tireoidianas em funcionários da UFRJ e estudo imunogenético dos indivíduos com anticorpos antitireoidianos positivos [tese de doutorado]. Rio de Janeiro: UFRJ, 2001. p. 82. Faculdade de Medicina - Universidade Federal do Rio de Janeiro, Rio de Janeiro, 2001.

5. Silva P, Romano A, Yazbek P Jr, Cordeiro J, Battistella L. Ergoespirometria computadorizada ou calorimetria indireta: um método não invasivo de crescente valorização na avaliação cardiorespiratória ao exercício. Rev Bra Med Esporte 1998;4/5:147-58.

6. Caraccio N, Natali A, Sironi A, Baldi S, Frascerra S, Dardano $A$, et al. Muscle metabolism and exercise tolerance in subclinical hypothyroidism: a controlled trial of levothyroxine. J Clin Endocrinol Metab 2005;90/7:4057-62.

7. Kahaly G. Cardiovascular and atherogenic aspects of subclinical hypothyroidism. Thyroid 2000;10:665-79.

8. American College of Sports Medicine. Diretrizes do ACSM para os testes de esforço e sua prescrição. $6^{a}$ ed. Rio de Janeiro: Guanabara Koogan, 2003.

9. Wasserman K, Hansen J, Sue DY, Whipp BJ. Principles of exercise testing and interpretation. Philadelphia: Lea \& Febiger, 1987.

10. Araújo CGS. Ergometria e ergoespirometria - algumas considerações. In: Rocha ML. Aspectos Diversos da Medicina do Exercício. 1ํe․ Rio de Janeiro: Revinter, 2004.

11. Yazbek P Jr, Carvalho RT, Sabbag LM, Battistella LR. Ergoespirometria. Teste de esforço cárdio-pulmonar, metodologia e interpretação. Arq Bras Cardiol 1998;71/5:719-24.

12. Oliveira FP, Giannella A. Metabolismo do oxigênio no exercício. In: David CM. Ventilação Mecânica - da fisiologia à prática clínica. Rio de Janeiro: Revinter, 2001. pp. 163-76.

13. Araújo CGS. Importância da ergoespirometria na prescrição de exercício ao cardiopata. SOCERJ 1998;XI/1(Jan/Fev/Mar).

14. Romaldini JH, Sgarbi JA, Farah CS. Disfunções mínimas da tiróide: Hipotiroidismo subclínico e hipertiroidismo subclínico. Arq Bras Endocrinol Metab 2004;48/1:147-58.

15. Kahaly GJ, Dillmann WH. Thyroid hormone action in the heart. Endocr Rev 2005;26/5:704-28.

16. Biondi B, Fazio S, Palmieri E, Carella C, Panza N, Cittadini A, et al. Left ventricular diastolic dysfunction in patients with subclinical hypothyroidism J Clin Endocrinol Metab 1999;84/6:2064-7.

17. Biondi B, Klein I. Hypothyroidism as a risk factor for cardiovascular disease. Endocrine 2004;24/1:1-13.

18. Dagre A, Lekakis J, Papaioannou T, Papamichael C, Koutras $D$, Stamatelopoulos $S$, et al. Arterial stiffness is increased in subjects with hypothyroidism. Int J Cardiol 2005;103:1-6.

19. Hamano K, Inoue M. Increased risk for atherosclerosis estimated by pulse wave velocity in hipothyroidism and its reversal with appropriate thyroxine treatment. Endocr J 2005;52/1:95-101.

20. Monzani F, Di Bello V, Caraccio N, Bertini A, Giorgi D, Giusti C, et al. Effect of levothyroxine on cardiac function and structure in subclinical hypothyroidism: a double blind, placebo-controlled study. J Clin Endocrinol Metab 2001;86/3:1110-5.

21. Noble BJ, Borg GVA, Jacobs I, Ceci R, Kaiser P. A categoryratio perceived exertion scale: Relationship to blood and muscle lactates and heart rate. Med Sci Sports Exerc 1983; 15:523-28.

\section{Endereço para correspondência:}

Míriam Raquel Meira Mainenti

EEFD - Labofise

Av. Pau Brasil 540

21949-900 Rio de Janeiro, RJ

E-mail: miriam.mainenti@hotmail.com 